\title{
W AND Z PRODUCTION: CROSS-SECTIONS AND ASYMMETRIES
}

\author{
M. WIELERS \\ Rutherford Appleton Laboratory, Chilton, Didcot, Oxon. OX11OER, United Kingdom \\ *E-mail: Monika.Wielers@cern.ch
}

\begin{abstract}
The LHC will be a $\mathrm{W}$ and $\mathrm{Z}$ factory. At nominal low luminosity of $\mathrm{L}=10^{33} \mathrm{~cm}^{-2} \mathrm{~s}^{-1}$ approx 15 $\mathrm{W} \rightarrow \mathrm{l} \nu$ events and $1.5 \mathrm{Z} \rightarrow \mathrm{ll}, \mathrm{l}=\mu$,e events will be produced per second. These processes will be important to test the Standard Model via precision measurements. W and Z production represent backgrounds to other Standard Model processes and signals of new physics and thus need to be understood precisely in the new kinematic region available at the LHC. In addition, they will be crucial physics channels to understand the detectors and its performances at start-up.
\end{abstract}

Keywords: Standard Model; W/Z production; ATLAS; CMS

\section{Introduction}

At the Large Hadron Collider (LHC) protons will be collided at a centre of mass energy of $14 \mathrm{TeV}$. The first collisions are expected in November 2007 at a centre of mass energy of $900 \mathrm{GeV}$. Collisions with $7 \mathrm{TeV}$ energy per proton beams are expected in the second half of 2008. The luminosity is expected to increase gradually up to the nominal low luminosity of $\mathrm{L}=10^{33} \mathrm{~cm}^{-2} \mathrm{~s}^{-1}$. For the first physics run the goal is to collect an integrated luminosity of several $\mathrm{fb}^{-1}$.

At the LHC two general multi-purpose detectors will be installed: CMS ${ }^{1}$ and ATLAS ${ }^{2}$. Both detectors have hadronic coverage up to pseudo-rapidities of $|\eta| \approx 5$. They are equipped to do precision physics in $|\eta|<2.5$. The cross section for the processes $\mathrm{W} \rightarrow \mathrm{l} \nu$ and $\mathrm{Z} \rightarrow \mathrm{ll}, \mathrm{l}=\mu, \mathrm{e}$ are approximately $15 \mathrm{nb}$ and $1.5 \mathrm{nb}$ respectively at the LHC. For an integrated luminosity of $1 \mathrm{fb}^{-1}$ around $10^{7}$ $\mathrm{W} \rightarrow \mathrm{e} \nu$ and $10^{6} \mathrm{Z} \rightarrow \mathrm{ll}$ events will be produced. At leading order (LO) W and Z production occur by the process $q \bar{q} \rightarrow W / Z$. At central rapidity $(|y|<2.5)$, the participating partons have small momentum fractions of typically $10^{-4}<x<0.1$ and therefore, the interaction happens mostly between sea quarks. The high scale of the process $Q^{2}=M^{2} 10^{4} \mathrm{GeV}^{2}$ implies the gluon is the dominant parton, thus, these sea quarks have mostly been generated by the flavour blind $g \rightarrow q \bar{q}$ splitting process.

$\mathrm{W}$ and $\mathrm{Z}$ events are useful for many purposes. On the technical side it will be used for a precise luminosity monitor, as high statistics detector calibration and alignment tool and to demonstrate the performances of the ATLAS and CMS detectors. On the physics side, they will allow testing the Standard Model (SM) via precision measurements. The following measurements are foreseen:

- Measurement of the inclusive $\mathrm{W}$ and $\mathrm{Z}$ cross-sections and the $\mathrm{W}+$ jet and $\mathrm{Z}+$ jet cross-sections.

- Constraining parton density functions (PDF's) using W events.

- Measurement of high-mass lepton par production.

- Precision measurements of the $\mathrm{W}$ mass and width ${ }^{3}$.

- Precise measurement of the weak mixing angle $\sin ^{2} \theta$.

- Measurement of gauge boson pair production and subsequently the measurement of the triple gauge couplings ${ }^{4}$. 


\section{Measurements of the $\mathrm{W}$ and $\mathrm{Z}$ cross sections}

The measurement of the $\mathrm{W}$ and $\mathrm{Z}$ cross sections will be one of the first measurements to be performed at the LHC when running at $14 \mathrm{TeV}$ centre of mass energy. W's and Z's will be reconstructed using their decays into electrons and muons. Studies have been performed based on Monte-Carlo (MC) samples including realistic detector simulations in ATLAS and CMS. Robust criteria have been developed which allow for a low-background event selection which is rather insensitive to detector inhomogeneities. Assuming $\int L d t=$ $1 \mathrm{fb}^{-1}$ the cross section measurement for $\mathrm{CMS}$ is $\sigma(p p \rightarrow Z+X \rightarrow \mu \mu+X)=$ $1160 \pm 1.5$ (stat) \pm 27 (syst) \pm 116 (lumi) pb and $\sigma(p p \rightarrow W+X \rightarrow \mu \nu+X)=14700 \pm$ 6 (stat) \pm 485 (syst) \pm 1470 (lumi) pb. The central value is indicative and assumes a cross section of 1160 (14700) pb for $Z \rightarrow \mu \mu$ $(W \rightarrow e \nu)$ events in the acceptance region $|\eta|<2.5$. The luminosity determination represents the dominant uncertainty, which initially is expected to be around $10 \%$ and will eventually decrease to $5 \%$ using the $\mathrm{W}$ and $\mathrm{Z}$ events themselves. For $Z \rightarrow \mu \mu$, the main contributions of the systematic uncertainty of $2.3 \%$ arise from the muon $p_{T}$ uncertainties $(1.8 \%)$. Possible uncertainties in the description of the muon $p_{T}$ spectrum have been have been estimated from a comparison between LO and NLO simulations. Further major uncertainties come from the tracker efficiency determination $(1 \%)$ and the PDF uncertainty $(0.7 \%)$. For $W \rightarrow \mu \nu$ events the main systematic uncertainties are $p_{T}$ effects in $\mathrm{LO} / \mathrm{NLO}(2.3 \%)$, missing transverse energy calculation $(1.3 \%)$, trigger efficiency $(1 \%)$ and muon efficiency $(1 \%)$.

\section{Extraction of PDF's using $\mathrm{W} \rightarrow \mathrm{e} \nu$ events}

As discussed in section 2, the uncertainties on $\mathrm{W}$ and $\mathrm{Z}$ boson production cross sec- tions are dominated by the PDF uncertainties: the $\mathrm{Z}$ and $\mathrm{W}$ rapidity distributions are theoretically known to $\mathrm{NNLO}^{5}$, with residual scale dependence $<1 \%$. The PDF uncertainty for rapidities below 2.5, corresponding to $10^{-4}<x<10^{-1}$, is $8 \%^{6}$. As shown in section 2 the background contamination on $\mathrm{W}$ events can be reduced to around $1 \%$ and the experimental uncertainties are small. Figure 1 shows the PDF uncertainties are

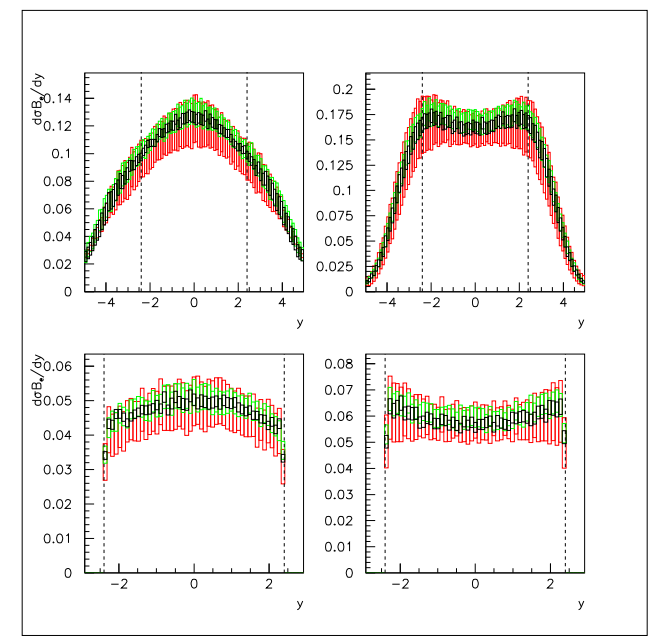

Fig. 1. Rapidity distributions of $e^{-}$(left plots) and $e^{+}$(right plots) with MRST2002 (black), CTEQ6.1M (dark grey) and ZEUS-S (light grey) and their quoted PDF uncertainties in $W \rightarrow e \nu_{e}$ events. At the top the distributions are shown at generator level at the bottom after application of the $W$ selection cut application using the fast ATLAS detector simulation program ATLFAST. The error boxes are the PDF uncertainties only. The dash vertical lines show the cuts at $|\eta|<2.4$.

only slightly degraded after detector simulation and selection cuts. Therefore, it will be possible to distinguish between different PDF sets looking at the lepton rapidity distribution in $W \rightarrow l \nu, l=e, \nu$ events. To estimate the contribution to a global PDF fit, ATLAS fully simulated pseudo-data has been included into the ZEUS-S PDF fit. Thus, the error on the low-x gluon shape parameter $\lambda$ $\left(x \cdot \mathrm{g}(x) \approx x^{-\lambda}\right)$ can be reduced by $40 \%$ using the data equivalent to few days of LHC 
running at $\mathrm{L}=10^{33} \mathrm{~cm}^{-2} \mathrm{~s}^{-1}$.

\section{Drell-Yan production}

In proton-proton collisions, the production of Drell-Yan (DY) pairs proceeds via s-channel exchange of photons and $\mathrm{Z}$ bosons. It starts from a combination of a valence quark and a sea quark (or a quark and an anti-quark from the sea) of the same flavour (e.g. $u \bar{u}, d \bar{d}$, etc). QCD effects enter the cross-section for DY production only in the initial state and thus make the predictions less uncertain. In the reaction $q \bar{q} \rightarrow l l$ only q can be a valence quark. Given the different average momentum fraction carried by valence and sea quarks, in most cases an asymmetric configuration will be preferred.

\subsection{High Mass Lepton Pair Production}

Measuring the lepton pair production will be an important benchmark process at the LHC as any deviation from the SM cross section can be interpreted as effects of a new physics signal. The most direct way is to look for effects of new physics as individual resonances above the Drell-Yan continuum. The experimental signature for DY events is distinctive: a pair of well isolated leptons with opposite charge. The backgrounds, which arise from leptons in $W^{+} W^{-}, \tau^{+} \tau^{-}, c \bar{c}, b \bar{b}, t \bar{t}$ fakes or cosmics are low. The cross-sections of Drell-Yan production and their statistical uncertainties for various integrated luminosities are shown in Table 1 using MC events fully simulated in the CMS detector. The systematic uncertainties are coming from the accuracy of theoretical calculations, accuracy of phenomenological determination of PDF's and the experimental uncertainties such as detector resolution, quality of fits, etc. The size of the uncertainties as a function of the di-muon pair invariant mass is summarised in Figure 2. More details can be found in ${ }^{1}$.
Table 1. Relative errors of the Drell-Yan muon pair cross-section measurements as found by CMS

\begin{tabular}{lcccc}
\hline $\begin{array}{l}M_{\mu \mu} \\
\mathrm{GeV}\end{array}$ & $\begin{array}{c}\sigma\left(M_{\mu \mu}\right) \\
\mathrm{fb}\end{array}$ & $\begin{array}{c}\text { Stat. } \\
1 \mathrm{fb}^{-1}\end{array}$ & $\begin{array}{c}\text { Stat. } \\
10 \mathrm{fb}^{-1}\end{array}$ & $\begin{array}{c}\text { Stat. } \\
100 \mathrm{fb}^{-1}\end{array}$ \\
\hline$\geq 200$ & $2.76 \cdot 10^{3}$ & 0.025 & 0.008 & 0.0026 \\
$\geq 500$ & $1.07 \cdot 10^{2}$ & 0.11 & 0.035 & 0.011 \\
$\geq 1000$ & 6.61 & 0.37 & 0.11 & 0.037 \\
$\geq 2000$ & $2.4 \cdot 10^{-1}$ & & 0.56 & 0.18 \\
$\geq 3000$ & $1.9 \cdot 10^{-2}$ & & & 0.64 \\
\hline
\end{tabular}

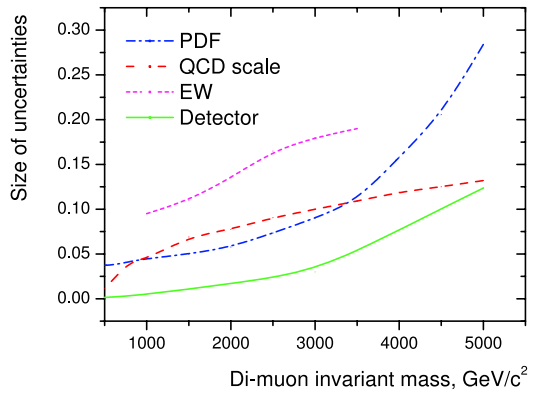

Fig. 2. Size of the electro-weak corrections and the cross section uncertainties from PDF's, hard process scale and detector performances as a function of the di-muon invariant mass cut.

\subsection{Measurement of $\sin ^{2} \theta_{W}$}

The forward-backward asymmetry is sensitive to new physics as well as to one of the fundamental parameters of the SM, the electroweak mixing angle $\sin ^{2} \theta_{W}$. This together with the top mass can subsequently be used to put constraints on the Higgs boson mass or, if the Higgs boson is discovered, will check the consistency of the SM.

To measure the forward-backward asymmetry, it is necessary to tag the directions of the incoming quark and anti-quark. At the LHC the initial state is symmetric. But in the reaction $q \bar{q} \rightarrow l^{+} l^{-}$only $q$ can be a valence quark, carrying on average a higher momentum compared to the sea anti-quarks. Therefore, $A_{F B}$ will be signed according to the sign of the rapidity of the lepton pair $y(l l)$. It increases as a function of $y(l l)$ (see Figure 3). The sensitivity of $A_{F B}$ to $\sin ^{2} \theta_{W}\left(M_{Z}^{2}\right)$ can be parametrised as $A_{F B}=$ 


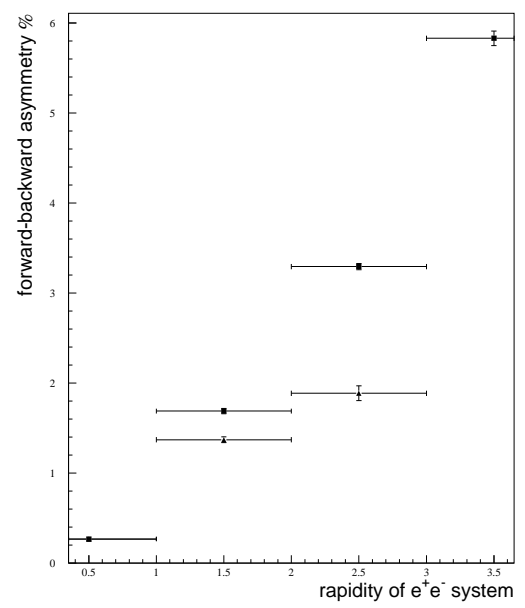

Fig. 3. Forward-backward asymmetry versus rapidity for $e^{+} e^{-}$pairs from $\mathrm{Z}$ decays satisfying the selection cuts $p_{T}(e)>20 \mathrm{GeV}, 85.2<M_{e e}<97.2 \mathrm{GeV}$. The asymmetry is shown where both electrons have $|y|<2.5$ (triangles) and where one electron is allowed to have $|y|<4.9$ (squares). The results are the same for both sets of cuts in the first bin.

$b \cdot\left(a-\sin ^{2} \theta_{W}\left(M_{Z}^{2}\right)\right)$. The values of $a$ and $b$ have been calculated in NLO QED and QCD. Studies have been carried out in the electron channel using the fast ATLAS simulation package. Efficiencies were taken using events fully simulated in the ATLAS detector. Two scenarios were considered: both electrons are identified in $|y|<2.5$ or in one electron is in $|y|<2.5$ and the other in $2.5<|y|<4.9$. The forward region is not covered by the tracking and electron identification is entirely based on calorimeter information, which allows for a moderate jet rejection. Figure 3 shows the measurement of $A_{F B}$ as a function of rapidity of the lepton pair for the two scenarios. For an integrated luminosity of $100 \mathrm{fb}^{-1}$ the statistical error ${ }^{7}$ of $\sin ^{2} \theta_{W}\left(M_{Z}^{2}\right)$ is $1.4 \cdot 10^{-4}$. Combining ATLAS and CMS measurements will decrease the error by $\sqrt{2}$. To exploit the possibility of measuring $\sin ^{2} \theta_{W}\left(M_{Z}^{2}\right)$ with such high precision, the systematic errors need to be comparably small. The most important ones are the uncertainty on PDF's and the precise knowledge of the lepton acceptance and efficiency.

\section{Summary}

At the LHC $\mathrm{W}$ and Z-bosons will be produced at a high rate. These processes are important means to test the Standard Model. One of the first measurements will be the measurement of the inclusive $\mathrm{W}$ and $\mathrm{Z}$ production cross sections and subsequently the $\mathrm{W}+$ jet and $\mathrm{Z}+$ jet cross sections. These measurements will demonstrate and require a systematic understanding of the detector performances (lepton identification, trigger efficiencies, etc.) and the backgrounds. It provides a test of (N)NLO perturbative QCD and helps constrain the PDF's, which is a main systematic uncertainty in many physics channels. High-mass lepton pair production will be an important benchmark process as any deviation from the SM crosssection will indicate new physics. Via measurement of the forward-backward asymmetry $\sin \theta_{W}\left(M_{Z}^{2}\right)$ can be precisely calculated. Together with the top mass the Higgs boson mass can be constrained or, if the Higgs boson is discovered, consistency checks of the SM will be carried out..

\section{References}

1. CMS Collaboration, in (CMS Technical Design Report, (CERN/LHCC 2006-21, CMS TDR 8.2, 2006).

2. ATLAS Collaboration, in (Detector and Physics Performance Technical Design Report, CERN/LHCC/99-14, 1999).

3. M. Malberti, $W$ mass and width at the $L H C$, these proceedings.

4. Z. Zhao, Studies of diboson productions and triple gauge coupling at the $\mathrm{LHC}$, these proceedings.

5. C. Anastasiou, L. Dixon, K. Melnikov, F. Petriello, (hep-ph/0312266, 2003).

6. A. Tricoli, A. Cooper-Sarkar, C. Gwenlan. (hep-ex/0509002, 2005).

7. G. Azuelos et al., in (hep-ph/003275, 2000). 